\title{
Técnicas de programación neurolingüística en la acción tutorial para potenciar el desempeño escolar
}

\author{
Techniques of Neurolinguistics Programming in the Tutorial Action to Enhance School \\ Performance
}

\section{Theira I. Samperio Monroy ${ }^{a}$, Jorge Martín Hernández Mendoza ${ }^{b}$, Laura Vallejo Hernández $^{c}$}

\begin{abstract}
:
At the higher level, the educational activity requires guidance and tutoring that allow students to become individuals with professional and human training that allows them to face any situation that life may pose. The tutorial task in our days requires the teaching commitment so that it is carried out efficiently and effectively, with a great human quality, to lead the students towards an assertive attitude. The management of neurolinguistic programming in educational aspects aims to offer the teacher tutor the necessary tools to increase the self-esteem of the students on which the achievement of their student goals and their self-realization as human beings depends, in order to contribute to their integral formation.
\end{abstract}

Keywords:

Neuro-linguistic programming, tutoring, school performance

\section{Resumen:}

En el nivel superior la actividad educativa requiere de orientación y tutoría que permitan que los estudiantes lleguen ser individuos con preparación profesional y humana que les permita enfrentarse a cualquier situación que la vida les pueda plantear. El quehacer tutorial en nuestros días requiere del compromiso docente para que se realice de forma eficaz y eficiente, con una gran calidad humana, para conducir a los estudiantes hacia una actitud asertiva. El manejo de la programación neurolingüística en aspectos educativos tiene como objetivo ofrecer al docente tutor las herramientas necesarias para aumentar la autoestima de los estudiantes de la cual depende el logro de sus metas estudiantiles y su autorrealización como seres humanos, para así contribuir a su formación integral.

Palabras Clave:

Programación neurolingúistica, tutoría, desempeño escolar

\section{Introducción}

La educación superior actualmente está presentando una transformación latente en los paradigmas de formación hacia los estudiantes, donde la atención personalizada puede ayudar a abatir los índices de reprobación y deserción escolar, así como a mejorar la eficiencia terminal [1].
La tutoría en el nivel superior pretende orientar y dar seguimiento al desarrollo de los estudiantes; apoyarlos en los aspectos cognitivos y afectivos del aprendizaje; fomentar su capacidad crítica y creadora y su rendimiento académico, así como perfeccionar su evolución social y personal, por ello se considera como un elemento estratégico para potenciar los esfuerzos institucionales orientados a mejorar la calidad educativa, la pertinencia social y la equidad.

\footnotetext{
a Profesor-Asignatura del Instituto de Ciencias Básicas e Ingeniería, Universidad Autónoma del Estado de Hidalgo, Área académica de computación y electrónica- Carretera Pachuca-Tulancingo km 4.5 Mineral de la Reforma Hidalgo, C.P. 42090. https://orcid.org/ 0000-00026944-7877, Email: smtheira@ hotmail.com

${ }^{\mathrm{b}}$ Profesor Tiempo Completo de la Escuela Superior de Tepeji del Río. Universidad Autónoma del Estado de Hidalgo, Av. del Maestro \#41 Col. Noxtongo 2da Sección, C.P. 42854. https://orcid.org/0000-0002-9262-4712, Email: jomar_hm@ @otmail.com
} 
El quehacer tutorial en las instituciones de educación superior debe aprovechar la tecnología de vanguardia para ofrecer estrategias innovadoras en las diversas actividades que se realizan en la tutoría para garantizar los buenos resultados en la vida académica de los estudiantes.

La Programación Neurolingüística pretende la mejora del proceso enseñanza aprendizaje, a través de la comunicación eficaz entre el docente y el alumno a partir de la generación de estados mentales estimulantes, lo cual ha ayudado a los docentes a eliminar viejos modelos que generaban miedo, trabas y bloqueos a los estudiantes y que dificultaban su aprendizaje.

Trabajar con técnicas de PNL en la práctica tutorial permite proporcionar las herramientas básicas para trabajar en la motivación del estudiante, su integración a la vida universitaria, desarrollar habilidades de estudio, apoyar y retroalimentar el proceso educativo, así como la orientación académica.

De igual forma, el Modelo Educativo de la Universidad Autónoma del Estado de Hidalgo (UAEH), ofrece a los estudiantes diferentes servicios de tutoría escolar como apoyo en su desempeño educativo, tanto a alumnos de bajo nivel de aprovechamiento como de alto desempeño [10]. En el presente trabajo de investigación se muestra la importancia de trabajar con diferentes técnicas de PNL en la acción tutorial con estudiantes del nivel superior, en la Licenciatura en Ciencias Computacionales de la Universidad Autónoma del Estado de Hidalgo, como una alternativa para potenciar el desempeño escolar y así disminuir los índices de deserción [8].

\section{Programa de Acción Tutorial de la UAEH}

La Universidad Autónoma del Estado de Hidalgo cuenta con un Programa Institucional de Tutorías, enfocándose al desempeño académico de los alumnos, fortaleciendo la toma de decisiones de éstos, a partir de la orientación y apoyo que le brinda el maestro tutor, con acciones específicas como son la tutoría individual y/o grupal, el interactuar en forma presencial $y / 0$ virtual, con la incorporación de alumnos tutores que ofrezcan apoyo y asesoramiento a sus compañeros, respaldados en un sistema de información y evaluación que retroalimente la actividad tutorial que se realiza en la institución y al mismo tiempo, permita conocer el impacto que el programa tiene en los índices de retención, aprobación y titulación [11].

Este Programa Institucional de Tutorías tiene una modalidad de registro en línea, que facilita la coordinación y organización de la acción tutorial en conjunto con los coordinadores tutores de las escuelas e institutos mediante reuniones de trabajo, visitas a sus instalaciones, así como una comunicación permanente que favorezca el proceso de las funciones [12].

\section{Definición de Programación Neurolingüística (PNL)} De acuerdo con Steve Bavister y Amanda Vickers, la Programación Neurolingüística se define como un modelo de comunicación que se centra en identificar y usar modelos de pensamiento que influyen sobre el comportamiento de una persona como una manera de mejorar la calidad y la efectividad de la vida $[3,4]$.

En el contexto educativo, la PNL propone mejorar la calidad del proceso de enseñanza aprendizaje, por medio de la comunicación eficaz entre docente y alumno, además de permitir generar estados mentales estimulantes para su logro [13].

\section{Técnicas de PNL en la educación}

Es importante destacar diferentes técnicas transformadoras de PNL útiles en la actividad tutorial:

1. Su modalidades o estilos de aprendizaje

2. Técnica de anclaje

3. Reencuadre

4. Calibración

5. Modelaje

6. Inducción

7. Sincronización

8. Relajación

Las técnicas de PNL, llevadas a cabo apropiadamente, son muy eficaces en cualquier ámbito, por ello es que son tan utilizadas en la actualidad. Aunque es importante mencionar que no es necesario aplicar todas en su conjunto en todas las situaciones, su manejo depende de las condiciones que se presente, en nuestro caso, en el ambiente de aprendizaje [9]. Con estas técnicas de PNL se persigue una transformación del estudiante que eleve su autoestima, incremente su capacidad de aprendizaje y le permita mejorar sus relaciones con los demás [5].

\section{Resultados preliminares}

Actualmente, se realizan pruebas piloto con alumnos de licenciatura de la Universidad Autónoma del Estado de Hidalgo, bajo los siguientes datos metodológicos de la siguiente Tabla 1:

\begin{tabular}{|l|l|}
\hline \multicolumn{1}{|c|}{ Características } & \multicolumn{1}{|c|}{ Encuesta } \\
\hline Población & $\begin{array}{l}\text { Docentes tutores y } \\
\text { estudiantes de la } \\
\text { licenciatura en ciencias } \\
\text { computacionales UAEH }\end{array}$ \\
\hline Ámbito de estudio & UAEH \\
\hline Unidad muestral & $\begin{array}{l}\text { Docentes, tutores y } \\
\text { estudiantes }\end{array}$ \\
\hline $\begin{array}{l}\text { Método de obtención de } \\
\text { información }\end{array}$ & \begin{tabular}{l} 
Aplicación de encuestas \\
\hline Tamaño de la muestra
\end{tabular} \\
\hline $\begin{array}{l}\text { Margen de error del } \\
\text { muestreo }\end{array}$ & $\begin{array}{l}5 \% \text { a un nivel global, para } \\
\text { un nivel de confianza del } \\
95 \%(p=q=0.5)\end{array}$ \\
\hline
\end{tabular}




\section{Fecha del trabajo de Febrero-junio 2018 campo}

Tabla 1. Metodología de investigación (elaboración propia)

Se tienen identificados a los estudiantes y docentes tutores que participarán en el estudio, a quienes ya se le aplicó la encuesta a fin de conocer su opinión acerca del manejo de técnicas de PNL en las actividades tutoriales.

Después del análisis preliminar de la información recabada, la cual se ha registrado en una base de datos, se puede mencionar que a la fecha se ha tenido una respuesta favorable en cuanto al conocimiento y manejo de técnicas de PNL en las actividades de tutoría durante el semestre, como se puede observar a continuación:

De los 50 estudiantes encuestados, 38 son hombres y 12 mujeres, y de los 20 docentes tutores encuestados 12 son hombres y 8 mujeres, lo que representa un mayor porcentaje de hombres en nuestro estudio. De esta muestra, el mayor rango de edad que hay entre los alumnos es de 19 a 30 años, mientras que la edad de los docentes oscila entre los 24 y 60 años. En general, el 74\% de los encuestados considera la acción tutorial muy relevante para garantizar el buen desempeño escolar. El $67 \%$ de los docentes tutores está dispuesto a implementar nuevas estrategias en las actividades tutoriales. El 73\% de los estudiantes está dispuesto a participar en las actividades que integran técnicas de PNL.

\section{Conclusiones}

En los contextos educativos de educación superior es importante estar analizando el impacto de la labor tutorial con respecto al rendimiento escolar de los alumnos, así como evaluar la eficiencia de la implementación de Programas Institucionales de Tutorías; lo anterior conlleva a implementar nuevas estrategias y técnicas que refuercen la actividad tutorial, como lo es el caso de las técnicas de PNL [6].

Las técnicas de PNL aplicadas en la acción tutorial en instituciones de educación superior permiten desarrollar diferentes competencias en los alumnos en su vida estudiantil y laboral, a través del trabajo en su motivación y autoestima [7].

A partir de los resultados preliminares, se puede observar que existe la necesidad de modificar los modelos educativos de acuerdo con las tendencias actuales, para que las estrategias pedagógicas de la práctica docente propicien ambientes adecuados para el aprendizaje efectivo de los estudiantes, en cuanto a conocimientos, actitudes y valores, donde la función del tutor pueda favorecer, en gran medida, el alcance de los objetivos propuestos.

Dado que el tutor es un formador en la vida educativa de los alumnos, ha resultado muy benéfico el manejo de técnicas de programación neurolingüística en la motivación de los estudiantes para potenciar su desempeño escolar, lo cual permite alcanzar las metas del Programa Institucional de Tutorías de la UAEH.

\section{Referencias}

[1] ANUIES. (2011). Asociación Nacional de Universidades e Instituciones de Educación Superior. México, D.F.: ANUIES. Recuperado el 28 de abril de 2018 desde http://www.anuies.mx/ [2] Silva G, Galeano E, Correa J. Compliance with the treatment. Acta Médica Colombiana 2005; 30(4): 268-73.

[2] ANUIES. (2011). Asociación Nacional de Universidades e Instituciones de Educación Superior. México, D.F.: ANUIES. Recuperado el 28 de abril de 2018 desde http://www.anuies.mx/

[10] UAEH. (2007). Manual del Tutor de la UAEH. Pachuca, Hgo., México: UAEH.

[8] Sambrano, J. (2000). PNL para todos. El modelo de la Excelencia. México. Editorial Alfaomega Grupo Editor.

[11] UAEH (2018). Dirección de Tutorías. Universidad Autónoma del Estado de Hidalgo. Recuperado el 29 de mayo de 2018 de https://www.uaeh.edu.mx/tutorias/

[12] UAEH (2013). Sistema de Información de Tutorías y Asesorías Académicas de la UAEH. Pachuca, Hgo., México: UAEH. Recuperado el 12 de abril de 2018 desde http://sistemas.uaeh.edu.mx/digse/tutoriasyasesorias/index.php

[3] Durán, B. (2018). Programación Neurolingüística (PNL): ¿qué es y cómo funciona? Psicología y mente. Recuperado el 2 de julio de 2018 de https://psicologiaymente.net/vida/programacion-neurolinguistica

[4] González Cano, M., Mendoza Austria, F. (2010). La tutoría en la formación profesional y empresa. Pachuca, Hgo., México: UAEH. Recuperado el 4 de mayo de 2018 desde http://www.uaeh.edu.mx/investigacion/icea/LI_GestEmp/marg_cano/5 .pdf

[13] UNAM (2013). La Programación Neurolingüística (PNL) mejora el proceso de enseñanza-aprendizaje. Fundación UNAM. Recuperado el 2 de mayo de 2018 de http://www.fundacionunam.org.mx/educacion/laprogramacion-neurolinguistica-pnl-mejora-el-proceso-de-ensenanzaaprendizaje/

[9] Sambrano, J. (2000). PNL para todos. El modelo de la Excelencia. México. Editorial Alfaomega Grupo Editor.

[5] Pastor, M. (2015). Las 8 estrategias transformadoras de la PNL. La mente es maravillosa. Recuperado el 15 de julio de 2018 de https://lamenteesmaravillosa.com/las-8-estrategias-transformadoras-la$\mathrm{pnl} /$

[6] Rodríguez Espinar, S. (2012). La acción tutorial para la Universidad. En S. Rodríguez (Eds.) Manual de Tutoría Universitaria. Recursos para la acción. Barcelona, España: Octaedro, Segunda Edición.

[7] Romo, A. (2011). La Tutoría. Una estrategia innovadora en el marco de los programas de atención a estudiantes. México, D.F.: Asociación Nacional de Universidades e Instituciones de Educación Superior, Dirección de Medios Editoriales. 